\title{
Videogame technology in Architecture Education
}

\author{
Francesc Valls ${ }^{1}$, Ernest Redondo ${ }^{1}$, David Fonseca ${ }^{2}$, Pilar Garcia-Almirall ${ }^{1}$, Jordi Subirós ${ }^{1}$ \\ ${ }^{1}$ Barcelona School of Architecture, UPC - BarcelonaTech, Barcelona, Spain \\ \{francesc.valls, ernesto.redondo, pilar.garcia-almirall\}@upc.edu, \\ jordi.subirosdestudiant.upc.edu \\ ${ }^{2}$ Architecture School, La Salle Universitat Ramon Llull, Barcelona, Spain \\ fonsiesalle.url.edu
}

\begin{abstract}
Videogame technology is quickly maturing and approaching levels of realism once reserved to $3 \mathrm{D}$ rendering applications used in architecture, in real-time and with the capacity to react in real-time to user input. This paper describes an educational experience using videogame technology in architecture education, exploring its applicability in the field in architecture compared to more traditional media. A prototype application modeling a proposed urban space was developed using Unreal Engine and a group of architecture students were asked to use the software to navigate the virtual environment. The development process of the applications is discussed as well as the design of the survey to assess the participants' experience in four key areas (a) player profile, (b) experience using the beta version, (c) use of videogame technology as an educational tool, and (d) applicability of game engines in Architecture.
\end{abstract}

Keywords: Gamification · Education $\cdot$ E-Learning $\cdot$ Architecture $\cdot$ Videogame Engine $\cdot$ Visual Representation · Interactivity $\cdot$ Immersion $\cdot$ Urban space

\section{Introduction}

Videogames are increasingly part of our life; while in the past playing videogames was considered an activity restricted to the young male demographic segment, the average age of a "gamer" (a person who plays videogames) is currently 35 years old and the gender distribution is remarkably balanced [1]. Moreover, the use of smartphones for leisure activities have widened the game-playing population spectrum while introducing new genres labeled as "social gaming" (games with emphasis on social interactions, usually with friends) and "casual gaming" (games designed to be played without needing special skills or strong player commitment, in contrast to "hardcore games").

The progressive importance of videogames in our lives is influencing our cultural expression; for example, Machinima Theater [2] uses videogames in performing arts, enacting classic plays using videogames, while indie (as in "independent") games [3] often use videogames to explore complex issues in their narrative [4], and can be a vehicle for expressing extremely deep emotions such as witnessing the suffering of a child with a medical condition [5].

Videogames have also been used in sports training, where a virtual partner increases the motivation of the subject while doing exercise in a virtual environment [6], and is beginning to find its place in the educational programs of Universities [7]. At the same time, the way games themselves are played is also evolving; the rise of livestreaming is influencing how games are designed and played [8], and game-playing is becoming a spectator sport [9].

\subsection{Videogames in educational contexts}

Gamification is the use of game mechanics in non-game situations [10], using the elements that make gameplaying engaging (e.g. competition, reward system, immersion) to improve learner motivation. On the other hand, serious games [11] simulate situations to practice specific skills, usually in training where practicing in a real setting is difficult, expensive or dangerous. Some educational experiences incorporate both gamification and serious games elements in different degrees.

The constructivist paradigm [12], where learners internalize new knowledge into their internal framework through accommodation (reframing existing knowledge to fit new one) and/or assimilation (incorporating new knowledge without altering existing one) has been applied in a gamified environment [13], where students produce in-game artifacts in a self-directed hands-on approach. Along these lines, the 
videogame Minecraft [14], and specifically its variant Minecraft Education [15], has been used as an educational tool [16], and the character from the TV series Doctor Who has been used in an online game to teach computer programming [17] to schoolchildren.

Other educational experiments confirm that using videogames in education can be a vehicle to promote reasoning in sciences [18], especially when using simulations [19, 20], and can be a valuable asset when used in e-learning [21], although some effort is necessary in its implementation to achieve the desired motivation [22]. Beyond school education, gamification has also been used in higher education [23] and workplace training [24], and videogames have even been used in machine learning to teach computers how to play a game [25], with a teacher agent instructing a student agent.

\subsection{Videogame technology in Architecture education}

Today, photorealistic synthetic imagery in architecture is almost undistinguishable from reality [26], but achieving comparable results in real-time is still challenging. The experience described in this paper describes a prototype exercise to test the applicability of teaching Architecture students using videogame technology.

The use of architecture-related content in videogames is not uncommon; beyond using the built environment as a background for the action taking place in a game, both at urban and architectural scales, we can find examples where elements of the architectural practice are central to the game mechanics, such as city-building games [27] which, beginning at the end of the 1980 decade with the SimCity [28] series to the more recent Cities Skylines [29], place the simulation of the life in a city as the core gameplay element. Beyond pure entertainment, the simulated environment of this game genre, although heavily simplified and mainly focused on mobility and infrastructure development [30], can help urban planners grasp basic concepts of their practice.

Regarding urban planning education, the Artificial Intelligence (AI) that power the agent-based models in the simulations of these games can be valuable in urban planning education [31], helping students assess the outcomes of their proposed policies in a "what-if" scenario, in a constructivist educational approach. In addition, three-dimensional geospatially referenced virtual environments [32] have also been proposed as an experimental approach to geographic analysis. These advances are made possible by advancements in 3D visualization [33] in Geographic information Systems (GIS), which in turn can benefit from the vast amount of urban data currently available [34].

Other experiences focus on the visual representation instead of the functional aspects of the simulation, and the realistic environments modern videogame engines are capable of producing have been used to explore the decision-making processes in landscape planning [35] and to support collaborative landscape planning [36].

Multiple teaching strategies can be used to deal with the complexity of education in the field of architecture, and the advantages of using 3D representations using Virtual Reality (VR) in contrast to the more traditional 2D plans in built environment education [37] can be beneficial, as well as using Augmented Reality (AR) to improve student engagement while promoting public participation [38].

\section{Objectives}

Architectural design has benefited from innovations in representation technologies, from the invention of conical perspective in the Renaissance to more recent introduction of Computer Aided Design (CAD) and, at the same time, these innovations have influenced directly or indirectly both the design workflow of the architectural practice and the formal language of the buildings.

This experience developed previous work by the authors [39], which proposed using videogames as an educational tool with two distinct strategies: (a) as a gamification experiment, through the incorporation of challenging and/or competitive elements to improve motivation, and (b) as a tool to promote critical thinking in architecture students placing them in simulated environments, to stimulate reflection on multiple aspects that are often overlooked in the design of public spaces, taking advantage of the Proteus effect [40] where self-representation in virtual worlds can affect the behavior of the participants.

The educational objective of the experience described in this paper was to test the applicability of using videogame engines in architecture education, specifically to research the students' perception on using this technology, and specifically to study if it could improve their motivation.

The application was an early beta prototype, following the software design philosophy of "release early, release often" [41] to enable a tighter feedback loop between the students and the instructors, which should 
be beneficial to both, producing better results that fulfill the requirements of the users while at the same time avoiding developing unneeded content.

In this version, the students' opinion was gathered through an online anonymous survey; in future iterations, they will be asked for permission to have their behavior logged in-game (using game analytics tools), and some elements of the survey will be incorporated into the executable.

As a secondary objective, the application laid the groundwork of a data-gathering tool to track the users' behavior in virtual space, to (a) find out if their behavior diverges from the behavior observed in a real (non-simulated) environments, and (b) to be used as a tool to perform controlled experiments, manipulating the simulated environment (i.e. lighting intensity, simulating disabilities, placing the user in crowded situations using AI-controlled avatars) and observing the changes in the behavior of the test subjects.

\section{Prototype development}

\subsection{Case of study}

The simulated environment was in a lot next to the building of the Barcelona School of Architecture of the Technical University of Catalonia (BarcelonaTech). This lot is currently the roof of an underground facility connected to the Engineering School of the same campus. The working premise was that this roof would be transformed into a public space, which would give access to the two towers of the Engineering School, that would be repurposed as multiple-use buildings with student dormitories and related facilities for the hosted students as well as other students in the campus (laundry, meeting rooms, convenience stores, etc.).

The site location was chosen because it was easy to visit by the architecture students, and allowed the comparison between the simulated proposed environment and the real built environment easily. In addition, it was expected that the familiarity with the site would make possible to identify contextual elements (visual or audio cues) that should aid in the sensation of being in a specific place.

\subsection{Choice of game development platform}

A review of the leading game engines available, considering three main factors (ease of development, feature set and licensing requirements) resulted in two main choices: Unity 5 [42] and Unreal Engine 4 [43]. The platform of choice was Unreal Engine 4.10 (UE) over Unity because it seemed more adequate for the purposes of the application to be developed. The main reasons of this choice were:

- While the new Unity version 5 supported Physically-Based Rendering (PBR), UE allowed programming complex shaders in a visual node-based editor like other 3D modeling and rendering packages commonly used in Architecture

- Most of the functionality needed for the application was built-in in UE (navigation, behavior trees, nodebased scripting) and therefore more tightly integrated than Unity, which relied on third-party solutions

- Both platforms had a marketplace with downloadable free and paid content, and while Unity had more content at the time of writing, the UE marketplace content was sufficient

- UE was free [44] for Architecture Visualization (ArchViz)

- The development language in UE was either Blueprint (node base scripting) or $\mathrm{C}++$, instead of $\mathrm{C} \#$ and UnityScript, which arguably allowed non-programmers access to advanced scripting

- Both platforms had a strong user community, and good documentation and training materials, and therefore this factor was not factored in the decision

Overall, it seemed that UE was more artist-friendly and less a developer tool, and could eventually be taught to Architecture students. However, the tool could actually have been developed in Unity as well, and newer versions of the application might use this platform instead.

\subsection{Rebuilding 3D assets}

Based on a SketchUp Model developed by the students on another course, the 3D assets had to be remodeled in a 3D package, because the SketchUp model was not ready to be used in the game engine: (a) the geometry was not optimized (it contained too many triangles), (b) many of the elements (i.e. windows, pillars) were repetitive and therefore there was a lot of potential for asset reusability, (c) the objects were not UV unwrapped, and (d) materials were not assigned to faces. 
The modeling phase involved breaking the geometry into simple objects that could be reused: the objects were remodeled with their geometry optimized, using multiple Levels of Detail (LOD) when necessary and given sensible pivot points (local transform), unnecessary non-visible faces were removed (since UE works with single-sided faces), normal maps were baked onto simplified geometry to simulate detail without increasing triangle count, materials were assigned to faces, objects were unwrapped using two UV channels (one for textures, and a second non-overlapping UV for lightmaps), hard and soft edges were defined, custom colliders were created when necessary, and finally the objects were exported to FBX files (the 3D asset file interchange format used by UE).

This way the geometry could be reduced from one $800 \mathrm{MB}$ FBX file to 55 FBX files with a combined size of less than $1 \mathrm{MB}$. These $3 \mathrm{D}$ assets were the building blocks of the simulated environment.

\subsection{Environment setup}

The environment design consisted in the following phases: (a) inserting the 3D objects into the scene, (b) defining the materials, (c) lighting setup, (d) reflection environment setup, and (e) visual effects.

The $3 \mathrm{D}$ objects were inserted into the scene using the UE editor. For repetitive assets, a custom construction script procedurally created a 3D array of instances of the meshes with definable number of copies and separation intervals for the $\mathrm{X}, \mathrm{Y}$ and $\mathrm{Z}$ axes. In addition, a class system was defined to help creating variants of compound objects (modules made of multiple 3D assets) to take advantage of inheritance from a parent class, adding or removing components when necessary.

The material setup consisted in assigning materials to the corresponding parts of the objects and defining the shaders and the texture assets each material should use. Instanced materials were defined to reuse the same material definition while allowing to change the scale of the object UV mapping to account for the different mesh dimensions.

Lighting was setup as two light sources, one directional light to account of sunlight and another skylight to account for the diffuse lighting from the sky. The lighting information (direct and indirect lighting, as well as shadows) was baked (pre-calculated) into lightmaps for all static (non-movable) objects in the environment.

Finally, some effects were added to improve immersion: eye adaptation (taking advantage of the High Dynamic Range (HDR) rendering of UE), bloom effects to compensate for the limited dynamic range of computer monitors when viewing very bright objects, flare effects to simulate the view from a physical camera, dynamic moving clouds in the sky dome, and reflections from the environment on materials such as glass (using spherical reflection probes).

\subsection{Avatar control definition}

The user avatar (pawn) inherited from the built-in character class in UE, adding a camera component at eye level, emulating a first-person perspective. Therefore, the pawn inherited the rigid body physics simulation functionality from the parent class and had the ability to collide with objects in the environment (using the capsule collider inherited from the character class) and climb stairs and ramps.

Since the movement functionality was inherited form the character parent class, the pawn had walk functionality and only the controls had to be defined. The controls replicated the standard First Person Shooter (FPS) functionality using the mouse and WASD keys:

- Moving forward/backwards: mapped to W and UP arrow (positive) and S and DOWN arrow (negative)

- Moving sideways: mapped to D and RIGHT arrow (positive) and D and LEFT arrow (negative)

- Turning the camera left/right: Mapped to horizontal movement of the mouse (X), controlling yaw (rotation around the vertical local axis of the avatar)

- Looking up/down: Mapped to vertical movement of the mouse (Y), controlling pitch (rotation around the left-right local axis of the avatar)

\section{Results}

The standard point of view when designing an architectural space is either a top-down perspective when working in 3D (either modeling on a computer or building a physical model) or a plan view when working in $2 \mathrm{D}$ (either in a CAD program or sketching freehand). Perspective representations are generated in a 


\section{uvvesstrat ramon LuU}

computer using a 3D rendering package or hand-drawn, but the results are either static images or noninteractive videos.

To find out the benefits that the use of game engines in architecture can provide to architectural visualization, focusing on (a) the use of a first-person perspective, and (b) the real-time interactivity, the resulting application was compiled into an executable and deployed to the students through the university Moodle-based intranet, where a Dropbox link was posted due to the maximum file size limitation of the platform. The students were asked to execute the application on their own computers and fill a survey after the experience.

On application launch, the avatar spawned facing the (virtual) building of the Barcelona School of Architecture to provide a familiar landmark to give the participants a contextual cue on the simulated environment. Therefore, the first-person camera was initially placed looking in the opposite direction of the place the students were expected to explore, to encourage them to walk around to get their bearings.

Placing the camera at the same eye-level as in the real world, added to the possibility of looking around emulating the head movement, was expected to mimic the sense of being in an architectural space more successfully than the traditional top-down view with orbit control commonly used in architecture, while providing a better sense of human scale (Fig. 1).

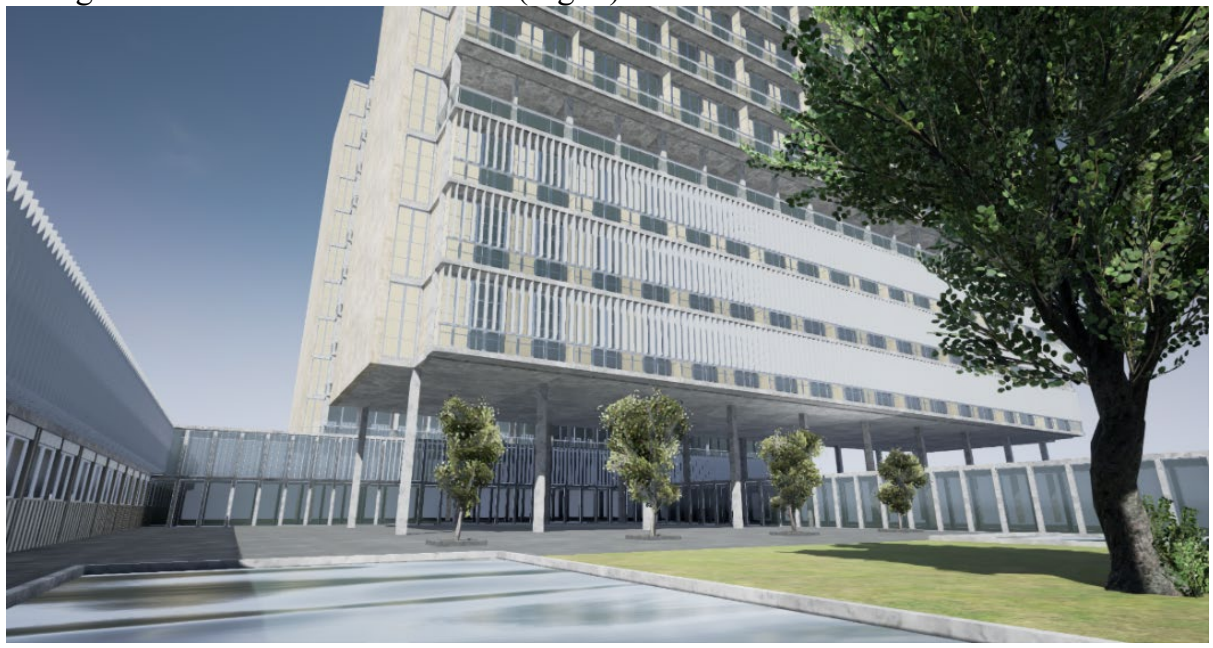

Fig. 1. In-game screenshots of the application visiting the virtual space

The participants were able to walk around the space, and had to navigate the environment avoiding obstacles (trees, pillars, buildings) and cross bridges over pools filled with water to reach some sections of the scene. The lighting when visiting the spaces underneath the buildings was dimmer and had to wait for their eyes to adapt to the relative darkness (Fig. 2).

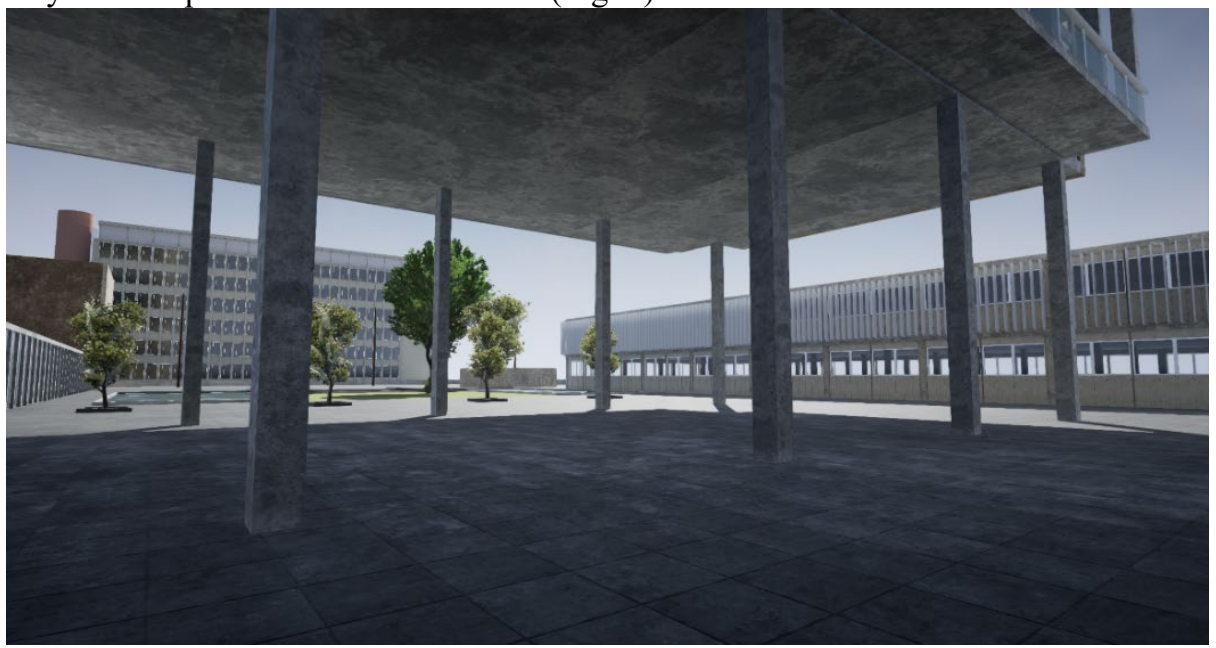

Fig. 2. In-game screenshots of the application featuring eye adaptation to dark environments 


\section{$5 \quad$ Survey design}

To gather data about their experience, the students were asked to fill a survey, using Google Forms. They were asked about four key aspects, using questions ranked in a 5-point Likert scale where applicable:

- Player profile

- Experience using the beta version

- Use of videogame technology as an educational tool

- Applicability of game engines in Architecture

The questions about the player profile tried to identify the motivation [45] of the participants and categorize them into six main profiles following a previous example of game motivation survey [46-48]: action, strategy, achievement, social, fantasy and creativity. The participants were also asked the average hours played daily in different platforms (computers, consoles and mobile devices) and their year of birth and gender.

The questions about the experience in the beta application asked about its usability (controls, speed and immersion), its graphics (materials, illumination, shadows, models, reflections and color) and the improvements that users would value in the next version of the software (more interaction, better visuals, sound cues, better sense of urban context, simulation of seasons or day/night cycles).

As and educational tool, the students were asked if the simulation promoted thinking differently about some aspects of the space, such as its size, proportions, materials or functionality.

Finally, the participants were asked about the applicability of game engines in architecture in different contexts (design, urban planning, visual representation, heritage) and to compare game engines to other technologies used by architects (rendering, Computer Aided Design, physical models, 3D printing, handdrawn sketches, photomontages, videos).

The analysis of the responses of the survey should give the authors insight on the participants' experience, which should improve the quality and usability of the next version of the program.

\section{Conclusions and future work}

The initial results show that students valued the experience of using videogame engines in architecture education, and the authors speculate that this acceptance will increase as engines improve and become easier to use. I this sense, in the 2015-16 academic year of the Architecture degree of the Barcelona School of Architecture, the students will be introduced in how to use UE in the context of the Multimedia course.

The next version of the application is expected to target HTML5 and run on modern browsers, to allow user participation over the Internet and increase the pool of participants while simplifying the distribution of the executable. This experience should be carefully designed taking advantage of gamification elements to increase involvement, to avoid participants dropping out of the experiment early, as some research suggests [49].

Actively manipulating the environment instead of just passively wandering in it should increase the engagement of the students because the resulting object will be built by themselves [50]. In the next iteration of the experience, it is planned to allow the participants not only to visit the virtual environment, but also interact with some elements, adding modular building blocks to create objects in-game which will allow the students to produce artifacts.

Finally, in future iterations of the experience, the changes in the perception of the space using realistic rendering compared to stylized (non-realistic) rendering [51, 52] will be assessed.

\subsection{Gathering experimental data in a virtual environment}

Tracking individual persons in public spaces is challenging; although it is possible to accurately track their movement using RFID or other technologies [53], it is technically difficult, and it raises privacy concerns when gathering data in public spaces. In areas such as economics, synthetic experiments are performed to model real situations, even though in some cases participants do not understand the rules fully [54].

The next phase of the development will include the capacity of tracking (with their consent) the users' behavior in virtual space (position and gaze along time), which will allow the application to be used as a research tool [55] to conduct controlled experiments. This experiments will focus on estimating movement parameters in pedestrian models [56], exploring wayfinding and search behavior [57], the mental representation of urban spaces [58, 59], as well as gaze behavior [60]. 
Acknowledgments. This research was supported by the Non-Oriented Fundamental Research Project EDU2012-37247/EDUC of the VI National Plan for Scientific Research, Development and Technological Innovation 2008-2011, Government of Spain, titled "E-learning 3.0 in the teaching of architecture. Case studies of educational research for the foreseeable future".

\section{References}

1. Entertainment Software Association: 2015 Essential Facts About the Computer and Video Game Industry. Entertainment Software Association (2015).

2. EK Theater: Retelling Classical Stories through Video Games, http://www.ektheater.com/.

3. Pajot, L., Swirsky, J.: Indie Game: The Movie. (2012).

4. Pope, L.: Papers, Please. 3909 LLC (2013).

5. Osit, D., Zouhali-Worrall, M.: Thank You for Playing. (2015).

6. Irwin, B.C., Scorniaenchi, J., Kerr, N.L., Eisenmann, J.C., Feltz, D.L.: Aerobic Exercise Is Promoted when Individual Performance Affects the Group: A Test of the Kohler Motivation Gain Effect. ann. behav. med. 44, 151-159 (2012).

7. Conditt, J.: "Citizen Kane" to "Call of Duty": The rise of video games in universities, http://www.engadget.com/2015/10/01/video-games-film-history-education-ashley-pinnick/.

8. Orland, K.: Twitch plays everything: How livestreaming is changing game design, http://arstechnica.com/gaming/2015/10/twitch-plays-everything-how-livestreaming-is-changing-game-design/.

9. Free to Play. (2014).

10. Deterding, S., Dixon, D., Khaled, R., Nacke, L.: From Game Design Elements to Gamefulness: Defining "Gamification." In: Proceedings of the 15th International Academic MindTrek Conference: Envisioning Future Media Environments. pp. 9-15. ACM, New York, NY, USA (2011).

11. Davidson, D. ed: Beyond Fun: Serious Games and Media. lulu.com, Pittsburgh (PA) (2010).

12. Papert, S.: Mindstorms: Children, Computers, and Powerful Ideas. Basic Books, Inc., New York, NY, USA (1980).

13. Weintrop, D., Holbert, N., Horn, M.S., Wilensky, U.: Computational Thinking in Constructionist Video Games. International Journal of Game-Based Learning. 6, 1-17 (2016).

14. Persson, M.: Minecraft. Mojang (2011).

15. Microsoft: Minecraft Education. Microsoft (2016).

16. Stuart, K.: Minecraft Education Edition: why it's important for every fan of the game, http://www.theguardian.com/technology/2016/jan/22/minecraft-education-edition-why-its-important-for-everyfan-of-the-game, (2016).

17. BBC Learning: The Doctor and the Dalek, www.bbc.co.uk/cbbc/games/doctor-who-game.

18. Holbert, N.R., Wilensky, U.: Constructible Authentic Representations: Designing Video Games that Enable Players to Utilize Knowledge Developed In-Game to Reason About Science. Tech Know Learn. 19, 53-79 (2014).

19. Honey, M., Hilton, M.L. eds: Learning science through computer games and simulations. National Academies Press, Washington, D.C (2011).

20. Aldrich, C.: Simulations and the Future of Learning: An Innovative (and Perhaps Revolutionary) Approach to eLearning. Pfeiffer, San Francisco (2003).

21. Domínguez, A., Saenz-de-Navarrete, J., de-Marcos, L., Fernández-Sanz, L., Pagés, C., Martínez-Herráiz, J.-J.: Gamifying learning experiences: Practical implications and outcomes. Computers \& Education. 63, 380 - 392 (2013).

22. Villagrasa, S., Fonseca, D., Romo, M., Redondo, E.: GLABS: Gamification for learning management systems. In: 2014 9th Iberian Conference on Information Systems and Technologies (CISTI). pp. 1-7 (2014).

23. Iosup, A., Epema, D.: An Experience Report on Using Gamification in Technical Higher Education. In: Proceedings of the 45th ACM Technical Symposium on Computer Science Education. pp. 27-32. ACM, New York, NY, USA (2014).

24. Oscar Garcia-Panella, Anna Badia-Corrons, Emiliano Labrador-Ruiz, David Fonseca-Escudero: Pleasant Learning Experiences: Augmenting Knowledge through Games and Interaction. In: Jiyou Jia (ed.) Educational Stages and Interactive Learning: From Kindergarten to Workplace Training. pp. 369-386. IGI Global, Hershey, PA, USA (2012).

25. Taylor, M.E., Carboni, N., Fachantidis, A., Vlahavas, I., Torrey, L.: Reinforcement learning agents providing advice in complex video games. Connection Science. 26, 45-63 (2014).

26. Parkin, K.: Building 3D http://www.cgsociety.org/index.php/CGSFeatures/CGSFeatureSpecial/building_3d_with_ikea.

27. Moss, R.: From SimCity to, well, SimCity: The history of city-building games, http://arstechnica.com/gaming/2015/10/from-simcity-to-well-simcity-the-history-of-city-building-games/.

28. Wright, W.: SimCity. Maxis (1989).

29. Korppoo, K.: Cities: Skylines. Colossal Order (2015).

30. Goodwin, D.: 10 Things The "Cities: Skylines" Video Game Taught Us About Modern Urbanism, http://www.archdaily.com/619567/rebuilding-simcity-10-things-cities-skylines-says-about-modern-urbanism/. 
31. Hjorth, A., Wilensky, U.: Redesigning Your City - A Constructionist Environment for Urban Planning Education. Informatics in Education. 13, 197-208 (2014).

32. Lin, H., Batty, M., Jørgensen, S.E., Fu, B., Konecny, M., Voinov, A., Torrens, P., Lu, G., Zhu, A.-X., Wilson, J.P., Gong, J., Kolditz, O., Bandrova, T., Chen, M.: Virtual Environments Begin to Embrace Process-based Geographic Analysis. Trans. in GIS. 19, 493-498 (2015).

33. Yin, L.: Integrating 3D Visualization and GIS in Planning Education. Journal of Geography in Higher Education. 34, 419-438 (2010).

34. Valls Dalmau, F., Garcia-Almirall, P., Redondo Domínguez, E., Fonseca Escudero, D.: From Raw Data to Meaningful Information: A Representational Approach to Cadastral Databases in Relation to Urban Planning. Future Internet. 6, 612-639 (2014).

35. Bishop, I.D.: Landscape planning is not a game: Should it be? Landscape and Urban Planning. 100, 390-392 (2011).

36. Herwig, A., Paar, P.: Game Engines: Tools for Landscape Visualization and Planning? In: Buhmann, E., Nothhelfer, U.G., and Pietsch, M. (eds.) Trends in GIS and virtualization in environmental planning and design. Proceedings at Anhalt University of Applied Sciences 2002. Wichmann Verlag, Heidelberg (2002).

37. Horne, M., Thompson, E.M.: The Role of Virtual Reality in Built Environment Education. Journal for Education in the Built Environment. 3, 5-24 (2008).

38. Fonseca, D., Valls, F., Redondo, E., Villagrasa, S.: Informal interactions in 3D education: Citizenship participation and assessment of virtual urban proposals. Computers in Human Behavior. 55, Part A, 504-518 (2016).

39. Valls, F., Redondo, E., Fonseca, D.: E-Learning and Serious Games: New Trends in Architectural and Urban Design Education. In: Zaphiris, P. and Ioannou, A. (eds.) Learning and Collaboration Technologies. pp. 632-643. Springer International Publishing Switzerland, Los Angeles, CA, USA (2015).

40. Yee, N., Bailenson, J.: The Proteus Effect: The Effect of Transformed Self-Representation on Behavior. Human Communication Research. 33, 271-290 (2007).

41. Raymond, E.S.: The Cathedral \& the Bazaar: Musings on Linux and Open Source by an Accidental Revolutionary. O’Reilly Media (1999).

42. Unity Technologies: Unity 5 . Unity Technologies (2015).

43. Epic Games: Unreal Engine 4. Epic Games (2012).

44. Epic Games, Inc.: Unreal Engine End User License Agreement, https://www.unrealengine.com/eula.

45. Yee, N., Ducheneaut, N., Nelson, L.: Online Gaming Motivations Scale: Development and Validation. In: Proceedings of the SIGCHI Conference on Human Factors in Computing Systems. pp. 2803-2806. ACM, New York, NY, USA (2012).

46. Yee, N.: How We Created the Gamer Motivation Profile, http://quanticfoundry.com/2015/06/18/how-we-createdthe-gamer-motivation-profile/, (2015).

47. Yee, N.: How We Developed The Gamer Motivation Profile v2, http://quanticfoundry.com/2015/07/20/how-wedeveloped-the-gamer-motivation-profile-v2/, (2015).

48. Yee, N.: Most Popular Games by Gaming Motivations, http://quanticfoundry.com/2015/08/11/most-populargames-by-gaming-motivations/, (2015).

49. Sauermann, H., Franzoni, C.: Crowd science user contribution patterns and their implications. PNAS. 201408907 (2015).

50. Norton, M.I., Mochon, D., Ariely, D.: The IKEA effect: When labor leads to love. Journal of Consumer Psychology. 22, 453-460 (2012).

51. Sloan, P.-P.J., Martin, W., Gooch, A., Gooch, B.: The Lit Sphere: A Model for Capturing NPR Shading from Art. In: Proceedings of Graphics Interface 2001. pp. 143-150. Canadian Information Processing Society, Toronto, Ont., Canada, Canada (2001).

52. Grabli, S., Durand, F., Sillion, F.X.: Density measure for line-drawing simplification. In: 12th Pacific Conference on Computer Graphics and Applications, 2004. PG 2004. Proceedings. pp. 309-318 (2004).

53. Kravets, D.: How the NFL - not the NSA - is impacting data gathering well beyond the gridiron, $\mathrm{http} / / /$ arstechnica.com/tech-policy/2015/09/the-nfl-is-reshaping-the-surveillance-society-xbox-one-experienceand-gambling/.

54. Burton-Chellew, M.N., Mouden, C.E., West, S.A.: Conditional cooperation and confusion in public-goods experiments. PNAS. 113, 1291-1296 (2016).

55. Kuliga, S.F., Thrash, T., Dalton, R.C., Hölscher, C.: Virtual reality as an empirical research tool - Exploring user experience in a real building and a corresponding virtual model. Computers, Environment and Urban Systems. 54, 363-375 (2015).

56. Vafayi, K., Muntean, A., Corbetta, A.: Parameter estimation of social forces in pedestrian dynamics models via a probabilistic method. Mathematical Biosciences and Engineering. 12, 337-356 (2014).

57. Pingel, T.J., Schinazi, V.R.: The Relationship Between Scale and Strategy in Search-Based Wayfinding. Cartographic Perspectives. 0, 33-45 (2014).

58. Hölscher, C., Tenbrink, T., Wiener, J.M.: Would you follow your own route description? Cognitive strategies in urban route planning. Cognition. 121, 228-247 (2011).

59. Schinazi, V.R., Epstein, R.A.: Neural correlates of real-world route learning. NeuroImage. 53, $725-735$ (2010). 
60. Wiener, J.M., Hölscher, C., Büchner, S., Konieczny, L.: Gaze behaviour during space perception and spatial decision making. Psychological Research. 76, 713-29 (2012). 